\title{
A SUCCESSFUL PREGNANCY OUTCOME IN A UTERUS DIDELPHYS A RARITY BUT A REALITY - A CASE REPORT
}

Medempudi Bhavya Sruthi ${ }^{1}$, M. G. Dhanalakshmi², Pushpalatha ${ }^{3}$

HOW TO CITE THIS ARTICLE:

Medempudi Bhavya Sruthi, MG Dhanalakshmi, Pushpalatha. "A successful pregnancy outcome in a uterus didelphys a rarity but a reality - a case report". Journal of Evolution of Medical and Dental Sciences 2013; Vol2, Issue 38, September 23; Page: 7337-7340.

ABSTRACT: Uterus didelphys belongs to class iii of American society of mullerian anomalies. Incidence is about $1 / 3000$ women which accounts to 0.1 to $0.5 \%$ of total women. Congenital uterine anomalies result from the abnormal formation, fusion or resorption of the Mullerian ducts during fetal life. A uterus didelphys results when bilateral Mullerian Ducts do not fuse but develop side by side resulting in a double uterus.

Although pregnancies can occur in patients with Mullerian duct anomalies, most of them have been linked to infertility, recurrent pregnancy loss, pre term deliveries, fetal mal-presentations and other obstetrics complications, making successful pregnancy outcome a rare situation in this condition. Uterine anomalies with successful pregnancy outcomes are rare.

We report a case of successful pregnancy outcome in a case of uterus didelphys bicollis for its rarity.

CASE DESCRIPTION: 22-year-old fourth gravida with no live issues was referred at 32 weeks+1 day with a singleton gestation in one horn of a uterus didelphys. A diagnosis of IUGR with severe oligohydramnios was made outside.

Her first pregnancy was a missed abortion at 3 months of amenorrhea for which she underwent suction evacuation. The diagnosis of uterus didelphys was made during this procedure, and was mentioned in the discharge summary. Patient doesn't have any scan images, HSG reports. As the blood typing indicated Rh negativity, Anti-D injection was given.

Her second pregnancy was a pre term delivery at home at 7 months of amenorrhea. The fetus was born dead. Anti-D was not administered.

Her third pregnancy was a twin pregnancy, terminated at 20 weeks and 6 days as an USG diagnosis of omphalocele and meningomyelocele was made. Manual removal of placenta was done. Anti-D injection was administered.

She underwent infertility treatment for a year before conceiving for the fourth time. She was referred at 32 weeks due to complications of severe oligohydramnios (AFI 2), intra uterine growth restriction detected in the third trimester scan. Patient was planned for caesarean section after investigations and ultrasound scan as per vaginal examination was unfavourable. Non stress test indicated fetal distress \& hence the patient was shifted for an emergency Caeserean section after adequate counseling of the patient $\&$ her relatives.

On table uterus didelphys \& pregnancy in the left uterus was noted. The fetus was in a breech presentation. The decision to do a Classical caesarean section was made as the lower segment of the left pregnant uterus was very narrow. 
A preterm female child weighing 1660gms was delivered as breech with APGAR 7/10 8/10 followed by the placenta. There were no immediate post-operative complications for the mother like PPH or adherent placenta.

The baby was in the neonatal intensive care unit for pre term care and was on paladai feeds. Anti- D was given to the mother as baby blood group is positive. Both mother and child got discharged after 10 days.

Literature Review: The true incidence and prevalence of Mullerian duct anomalies in the general and in the infertile population is not accurately known. The incidence and prevalence of these anomalies vary widely. However, prevalence ranging from 0.16 to $10 \%$ has been reported. Int J Med Invest. 2(1):61-64: 2013 - Successful Consecutive Singleton Pregnancies In Separate Horns Of A Uterus Didelphys: A Case Report by Hüseyin Aksoy, Ülkü Aksoy, Gökhan Açmaz - Kayseri Military Hospital Department Of Obstetrics And Gynecology, Turkey

DISCUSSION: Congenital Mullerian duct defects are clinical problems encountered by Obstetricians \& Gynecologists. Uterine structural anomalies are often asymptomatic and normal pregnancies can occur in patients with Mullerian duct anomalies. These anomalies are often discovered during pregnancies or at the time of delivery and abortion or during infertility evaluation. Sometimes such anomalies are made out during a caesarean section or during laparoscopy/laparotomy. In our case uterus didelphys was diagnosed during suction evacuation done for her first pregnancy which was a missed abortion.

Comparing women with a normally shaped uterus, the women having any type of Mullerian duct anomalies must expect to have significantly higher risk of obstetric complications such as spontaneous abortion, recurrent pregnancy loss, premature labor, malpresentation and dystocia at delivery.

A study by Heinonen (2) (1984) reported a study in 26 women with uterine didelphys, out of which all 26 women had dysmenorrhoea, dyspareunia and other gynaecological symptoms. All patients had double vagina, fetal survival rate $67.5 \%$ breech $43 \%$, preterm delivery $21 \%$, IUGR $10 \%$, caesarian section $82 \%$. All 26 patients had a longitudinal vaginal septum, occasionally; one hemi vagina is obstructed by an oblique or transverse vaginal septum (3). Multifetal gestation is unusual in these women (4)

Majority of women with Mullerian duct anomalies have little problem conceiving, they have higher rates of spontaneous and recurrent abortions, as was noted in our patient. This report describes one case of a successful pregnancy outcome in a uterus didelphys. Our patient had successful pregnancy outcome in a uterus didelphys bicollis after three unsuccessful pregnancy outcomes with various complications of the uterine anomaly.

To our knowledge, the present case though a rarity, was a reality.

CONCLUSION: In conclusion, although frequently asymptomatic with possibility of normal pregnancies in patients with Mullerian duct anomalies, all of these congenital anomalies have been associated with infertility, recurrent pregnancy loss, preterm delivery, fetal malpresentation and other obstetric complications, all of which increase perinatal morbidity and mortality rates. 


\section{REFERENCES:}

1. Hüseyin Aksoy, Ülkü Aksoy, Gökhan Açmaz: Successful Consecutive Singleton Pregnancies In Separate Horns Of A Uterus Didelphys: A Case Report: Kayseri Military Hospital Department Of Obstetrics And Gynecology, Turkey Int J Med Invest. 2(1):61-64: 2013

2. Heinonen PK. Uterus didelphys: a case report of 26 cases. Eur J Obstet Gynecol Reprod Biol. 1984 Jul;17(5):345-50

3. Asha B, Manila K: An unusual presentation of uterus didelphys with obstructed hemivagina with ipsilateral renal agenesis. Fertil steril90 (3): 849. e9, 2008

4. Olah KS: uterine torsion and ischemia of one horn of a bicornuate uterus: a rare cause of failed second trimester termination of pregnancy. Br j obstet gynaecol52 (1):40, 2009
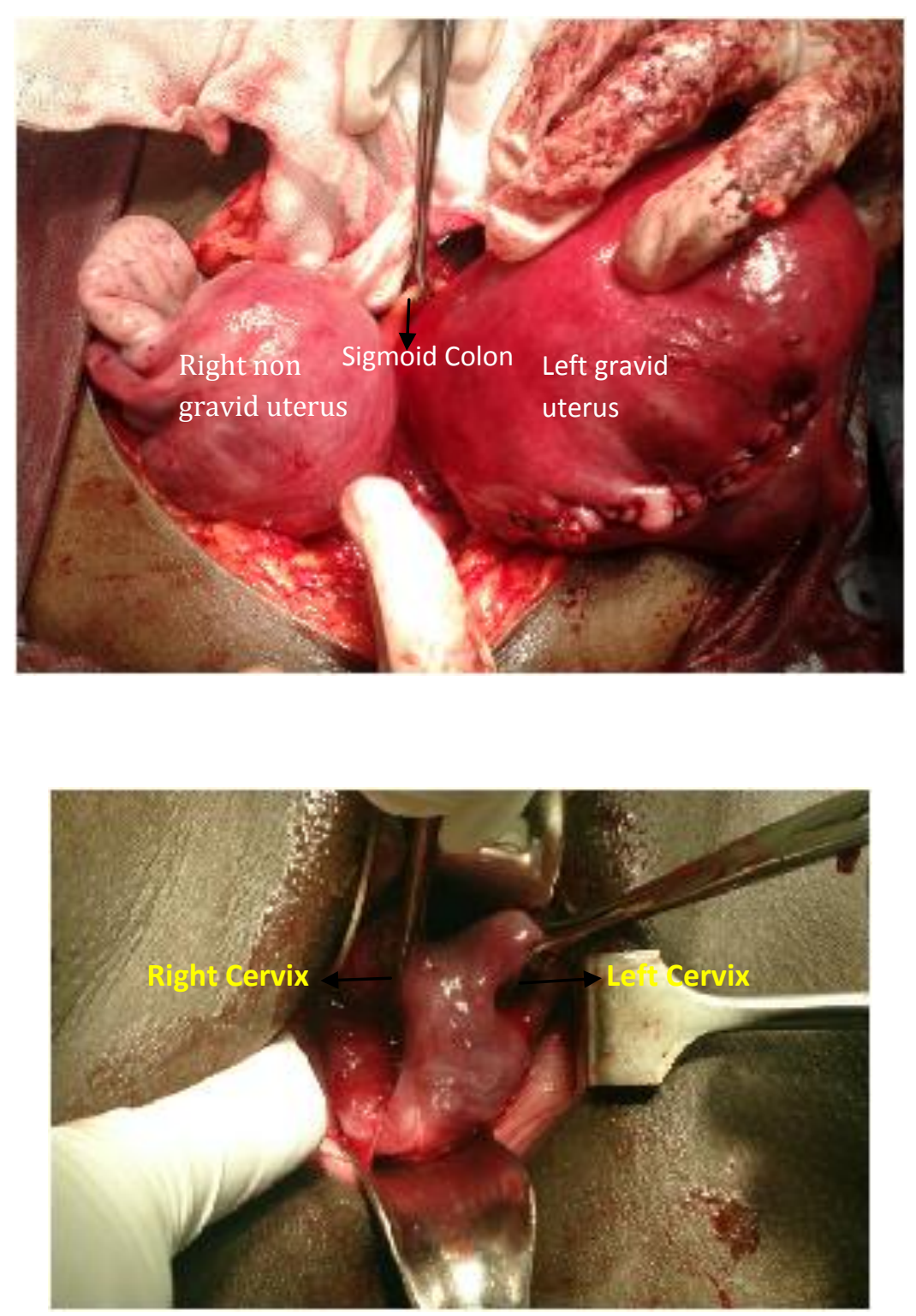


\section{AUTHORS:}

1. Medempudi Bhavya Sruthi

2. M.G. Dhanalakshmi

3. Pushpalatha

\section{PARTICULARS OF CONTRIBUTORS:}

1. Post Graduate, Department of Obstetrics \& Gynaecology, Sri Ramachandra Medical College.

2. Professor, Department of Obstetrics \& Gynaecology, Sri Ramachandra Medical College.

3. Professor, Department of Obstetrics \& Gynaecology, Sri Ramachandra Medical College.
NAME ADDRESS EMAIL ID OF THE

\section{CORRESPONDING AUTHOR:}

Dr. Medempudi Bhavya Sruthi, B-302, Jashine Court, Kattupakkam, Mount Poonhamalle Road, Chennai.

Email-mmascotmascot@gmail.com

Date of Submission: 05/09/2013. Date of Peer Review: 06/09/2013. Date of Acceptance: 14/09/2013.

Date of Publishing: 21/09/2013 